\title{
Beiträge zur Kenntniss des Strychnins
}

\author{
(I. Mittheilung) \\ von \\ Dr. Karl Garzarolli Edler v. Thurnlackh, \\ Privatdocent an der $k . k$. deutschen Tniversität in Prag.
}

(Vorgelegt in der Sitzung am 6. December 1888.)

Gelegentlich des Studiums einiger Reactionen von Alkylhalogenadditionsproducten des Strychnins kam ich in die Lage auch einige dieser Verbindungen herzustellen, welche bisher noch nicht bekannt gewesen sind. Hierzu gehören die im folgenden besehriebenen Benzylstrychniliumverbindungen. ${ }^{1}$

\section{Benzylstrychniliumchlorid.}

Sehr fein zerriebenes Strychnin, Sm $262^{\circ}$ (aus dem Nitrat durch Fällen mit Ammoniak und Umkrystallisiren des Niederschlages aus Alkohol dargestellt) wurde mit absolutem Alkohol aufgeschlämmt in eineın mit Rückflusskühler versehenen Kolben am Wasserbade gelinde erwärmt und zu demselben etwas mehr als die berechnete Menge Benzylchlorid hinzugefügt. Sehr bald trat eine heftige Reaction ein, welche die Bildung einer weissen, harten Masse zur Folge hatte.

Nun wurde soviel kochender Alkohol $(80 \%)$ zugefügt, bis die feste Substanz gelöst war, und die Flüssigkeit etwa acht Stunden hiedurch zam gelinden Sieden erhitzt.

1 Ich ziehe diese Bezeichnungsweise, die übrigens auch schon von anderen angewandt wurde, der üblichen Methyl-, $\ddot{A}$ thyl-, Benzylstrychnin vor, da sie dem Charakter der Verbindung entsprechender ist, als letztere, welche nur zur Bezeichnung von Strychninderivaten angewendet werden sollte, in denen ein an Kohlenstoff gebundenes Wasserstoffatom durch die. Methyl-, ̈̈thyl-, Benzylgruppe ersetzt worden ist.

Chemia-Heit N: 1. 
K. v. Garzarolli,

Nach dem Erkalten schieden sich dicke weisse Prismen ab; aus der von denselben befreiten Mutterlange wurde dureh weitere Concentration eine neue Krystallausscheidung gewonnen.

Diese Krystalle wurden nach dem Waschen mit Äther-Alkohol durch wiederholte Krystallisation aus kochendem Wasser gereinigt.

Das so gewonnene Chlorid stellt kurze, dicke Prismen, welche meist stark getrübt erscheinen, oder kleine glänzende Täfelchen dar, die sich in heissem Wasser ziemlich leicht, in kaltem und in Alkohol jedoch schwer lösen. Es enthält ein Moleküil Krystallwasser, welches bei $110^{\circ} \mathrm{C}$. vollständig abgegeben wird.

Das wasserfreie Salz schmilzt zwischen $262-263^{\circ}$ C. unter totaler Zersetzung.

I. $1.0078 g$ Substanz gaben bis zu $110^{\circ} \mathrm{C}$. erhitzt einen Gewichtsverlust von $0.0392 \mathrm{gr}$.

II. $0 \cdot 5703 g$ wasserhältiges Salz lieferten $0 \cdot 1670 g$ Chlorsilber. III. $0.2871 g$ der wasserfreien Verbindung gaben $0 \cdot 7614 g$ Kohlensäure und $0 \cdot 1719 \mathrm{~g}$ Wasser.

IV. $0.7639 \mathrm{~g}$ wasserfreier Substanz einer anderen Bereitung ergaben $0 \cdot 2360 \mathrm{~g}$ Chlorsilber.

Wasserbältiges Salz.

Gefunden:

I. $\mathrm{H}_{2} \mathrm{O} . .3 \cdot 89 \%$

II. Cl . . $7 \cdot 24 \%$

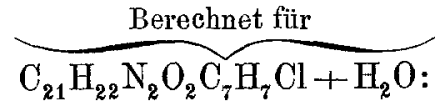

$\mathrm{H}_{2} \mathrm{O}$. . . $3 \cdot 75 \%$

$\mathrm{Cl}$

Wasserfreies Salz.

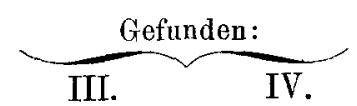

C. . $72 \cdot 33 \%$

H. . $6 \cdot 66^{\%} \%$

$\mathrm{Cl}$ Versuche über die physiologische Wirkung des Präparates anzustellen, verdanke ich folgende Mittheilung: 
„Nach den von mir angestellten Versuchen ist das Benzylstrychnin für Kalt- und Warmblütler ein heftiges Gift.

Frösche werden durch $2 m g r$ des Chlorids bei Einführung in den Rückenlymphsack in etwa 15 Minuten, durch circa $5 \mathrm{mgr}$ in 3 Minuten völlig gelähmt. Die Lähmung beruht vorwiegend, wie beim Curare, auf dem Unerregbarwerden der Nervenendigungen in den willkürlichen Muskeln.

Beim Kaninchen veranlassen 20-25 mgr subcutan injicirt erst Steigerung der Reflexe und an Strychnintetanus erinnernde Krämpfe, sodann rasch zunehmende Paralyse. Der Tod tritt ohne Krämpfe etwa in einer Stunde nach Beibringen der tödtlichen Dosis ein. Auch hier ist eine curareartige Wirkung leicht nachzuweisen."

\section{Benzylstrychniliumnitrat.}

Zur Herstellung dieses Salzes wurde das Chlorid in kochendem Wasser gelöst und mit der abgemessenen Menge einer Silbernitratlösung von bekanntem Gehalte zersetzt. Da das Nitrat sehr schwer löslich ist, so miissen die Lösungen sebr verdünnt angewendet und das Chlorsilber aus der siedend heissen Lösung abfiltrirt werden. Beim Abkühlen scheidet sich das Salz in glänzenden, kleinen Prismen, in Täfelchen, sehr häufig auch in Durchkreuzungszwillingen ab.

Die Verbindung ist wasserfrei.

Auf $240^{\circ}$ C. erhitzt färbt sie sich gelb, zwischen $262^{\circ}$ bis $265^{\circ} \mathrm{C}$. findet ein theilweises Schmelzen der Substanz statt, wobei sie sich jedoch unter Abspaltung rotber Dämpfe zersetzt.

$1.3502 g$ Substanz erlitten, bei $110^{\circ}$ bis zur Gewichtsconstanz erhitzt, einen Gewichtsverlust von $0.0024 \mathrm{~g}$. $0.3452 g r$. des Salzes gaben verbrannt $0 \cdot 8665 g$ Kohlensäure und $0 \cdot 1969 \mathrm{~g}$ Wasser.

\begin{tabular}{|c|c|c|}
\hline & Gefunden: & Berechnet für: \\
\hline & & $\mathrm{C}_{21} \mathrm{H}_{22} \mathrm{~N}_{2} \mathrm{O}_{2}\left(\mathrm{C}_{7} \mathrm{H}_{7}\right) \mathrm{NO}_{3}:$ \\
\hline &. $.68 \cdot 45 \%$ & C... $.68 \cdot 96 \%$ \\
\hline & . . $6 \cdot 35^{0} \%$ & $\mathrm{H} \cdot . \cdot 5 \cdot 97^{\circ} \%$ \\
\hline
\end{tabular}

\section{Benzylstrychniliumbichromat}

wurde durch Fällen einer Lösung des Chlorides mit einer Kaliumbichromatlösung als flockiger orangegelber Niederschlag erhalten. 
Nachdem er krystallinisch geworden war, wurde er von der Mutterlange abgesangt, mit kaltem Wasser ausgewaschen und aus kochendem, indem er übrigens auch sehr schwer löslich ist, umkrystallisirt.

Die ausgeschiedenen orangerothen Kryställchen erschienen unter dem Mikroskope als kurze schiefe Säulen, welche im Inneren treppenartige Absätze aufwiesen.

Die Krystalle sind wasserfrei.

$0 \cdot 2074 g$ Substanz gaben $0.0300 g$ Chromoxyd.

$0 \cdot 2622 g$ verbrannt gaben $0 \cdot 6020 \mathrm{gr}$. Kohlensäure und $0 \cdot 1374 \mathrm{~g}$.

Wasser.

$$
\begin{aligned}
& \text { Gefunden: } \\
& \text { C. . . } 62 \cdot 62 \% \\
& \mathrm{H} \text {. . . } 5 \cdot 84 \% \\
& \mathrm{Cr} . .9 \cdot 91 \%
\end{aligned}
$$

\section{Benzylstrychniliumsulfocyanid.}

Eine wässerige Lösung von Benzylstrichniliumchlorid wurde mit Sulfocyankalium versetzt, wodurch ein weisser, flockiger Niederschlag entstand, der in wenigen Stunden krystallinisch wurde. Derselbe wurde abgesaugt, ausgewaschen und aus viel kochendem Wasser krystallisirt.

Das Salz scheidet sich in Nadeln, die häufig zu Büscheln vereinigt, manchmal auch federfahnenartig angeordneterseheinen, aus. Es ist wasserfrei und schmilzt zwischen $236^{\circ}-237^{\circ} \mathrm{C}$. $0 \cdot 1410 \mathrm{~g}$ Substanz gaben $0 \cdot 3700 \mathrm{~g} \cdot \mathrm{K} n$ hlensäure und $0.0842 \mathrm{~g}$ Wasser.
Gefunden:
C... $71 \cdot 57 \%$
H. . . $6 \cdot 65 \%$
Berechnet für:

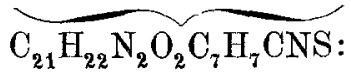
C... . $72 \cdot 09 \%$
$\mathrm{H}$. . . . $6 \cdot 02 \%$.

\section{Benzilstrychniliumchlorid-Platinchlorid}

wurde durch Mischen der Lösungen der beiden Componenten als ein fleischrother, amorpher Niederschlag erhalten. 
Da die mit dem gut ausgewaschenen Niederschlag angesteliten Krystallisationsversuche vergeblich waren, ja meist mit der Zersetzung der Substanz endigten, so wurde der noch vorhandene Rest bis zur Erlangung eines constanten Gewichtes getrocknet und zur Platinbestimmung benützt.

Die Verbindung schmilzt zwischen $215^{\circ}-216^{\circ} \mathrm{C}$. $0 \cdot 2270 \mathrm{~g}$ Substanz gaben $0 \cdot 0353 \mathrm{~g}$ Platin.

$$
\text { Pt. . . . } 15.58 \% \quad \frac{\text { Gefunden: }}{\begin{array}{c}
\left(\mathrm{C}_{21} \mathrm{H}_{22} \mathrm{~N}_{2} \mathrm{O}_{2} \mathrm{C}_{7} \mathrm{H}_{7} \mathrm{Cl}\right)_{2} \mathrm{PtCl}_{4} \\
\mathrm{Pt} . . .215 \cdot 49 \%
\end{array}}
$$

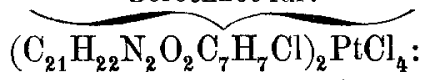

Um die freie Basis,

\section{das Benzylstrychnilinmhydroxyd}

darzustellen, wurde das feingepulverte Chlorid mit frisch gefälltem Silberoxyd zusammengerieben und die vom Niederschlage befreite, dunkel kirschrothe ${ }^{1}$ Lösung im Vacuum eingedampft.

Es schieden sich silbergrau gefärbte Nädelchen ab, welche aus kochendem Weingeist umkrystallisirt wurden. Da die erhaltenen Krystalle noch gefärbt waren, so wurden sie wieder in Alkohol gelöst und mit Äther versetzt. Die entstehende Fällung enthielt neben mit gerissener Basis den Farbstoff.

Durch Abdunsten der Flüssigkeit wurde das Benzylstrychniliumhydroxyd in seideglänzenden, blassrosenroth gefärbten Blättchen erhalten. Die schwach röthlich gefärbte Lösung reagirte stark alkalisch und fällte Eisenchloridlösung.

$0.1007 \mathrm{~g}$ bei $100^{\circ} \mathrm{C}$. getrockneter Substanz gaben $0.2829 \mathrm{~g}$ Kohlensäure und $0 \cdot 0633 g$ Wasser.
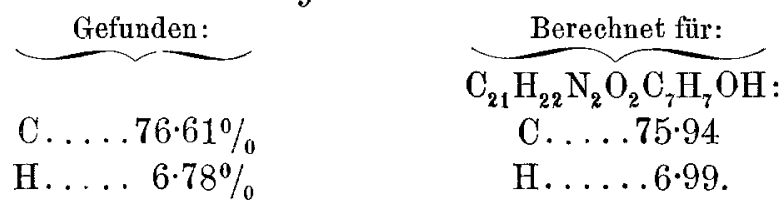

\section{Reactionen der Benzylstrychniliumverbindungen.}

Zur Ausführung derselben wurde eine bei gewöhnlicher Temperatur gesättigte Lösung des Chlorides benützt.

1 Die Färbung wird wahrscheinlich durch ein Oxydationsproduct der Base bewirkt. 
K. v. Garzarolli,

Concentrirte Kalilauge verursacht anfangs milchige Trübung, der bald, unter Klärung der Flïssigkeit, die Abscheidung feiner Nädelchen folgt. Zusatz von Wasser bewirkt Lösung des Niederschlages.

Natriumcarbonat erzengt keinen Niederschlag, nach längerem Stehen oder beim Erwärmen schwach röthliche Färbung.

Jod-Jodkaliumlösung fällt einen braunen amorphen Niederschlag.

Ferrocyankaliumlösung gibt einen lichtgelben, allmählich krystallinisch werdenden Niederschlag, der sich beim Erwärmen in der Flüssigkeit vollständig löst und später krystallinisch ausfällt.

Ferricyankaliumlösung erzeugt einen gelben Niederschlag, der sich beim Erwärmen theilweise löst (unverändert?), während ein anderer Theil schmilzt und eine harzige Masse gibt.

Salpetersäure fällt aus nicht zu sehr verdünnten Lösnngen das krystallinische Nitrat. Wird die Flüssigkeit gekocht, so färbt sie sich intensiv gelb.

Versetzt man in einem Porzellanschälchen einen Tropfen des Chlorides mit einem Tropfen concentrirter Schwefelsäure und lässt dann eine Spur einer Ferrocyankaliumlösung einfliessen, so bemerkt man die Bildung eines griingelb gefärbten Niederschlages, der an den Rändern violettroth gefärbt erscheint. Beim Verrïhren der Flüssigkeit tritt eine verschwindende blauviolette Färbung auf, die in Rosaroth übergeht. Dieser Farbenton hält sich stundenlang.

Ferricyankalium und Schwefelsäure bringen dieselbe Färbung bervor wie beim Strychnin.

Quecksilberchlorid fällt einen weissen amorphen, in viel kochendem Wasser löslichen Niederschlag, der beim Erkalten undeutlich krystallinisch ausgeschieden wird.

Jodwismuth-Jodkaliumlösung gibt einen orangerothen Niederschlag, welcher mikrokrystallinisch ist.

Gelbes Schwefelammon erzeugt einen gelben amorphen Niederschlag.

Die mit Kaliumbichromat, Kaliumsulfocyanid und Platinchlorid entstehenden Niederschläge wurden bereits erwähnt. 


\section{Oxydation des Benzylstrychniliumchlorids.}

$10 \mathrm{~g}$ der Verbindung wurden in warmem Wasser gelöst und mit $25 g$ Kaliumpermanganat (in $4 \%$ Lösung) versetzt. Die Oxydation erfolgte anfangs sehr rasch ${ }^{1}$, später verlangsamte sich dieselbe und musste unter Erwärmung auf circa $60^{\circ}$ C. zu Ende geführt werden.

Die über dem Mangansuperoxyde stehende farblose Flüssigkeit hatte sich nach etwa zwölfstündigem Stehen bräunlich gefärbt, sie wurde vom Niederschlage getrennt und dieser, nach dem Auswaschen mit kaltem Wasser, ausgepresst.

Filtrat und Waschwässer wurden vereinigt und concentrirt und die so erhaltene, dunkelbraun gefärbte, alkalisch reagirende Lösung solange mit Salzsäure versetzt, als noch Harzausscheidung erfolgte. Das vom Harze getrennte Filtrat wurde mit der Flüssigkeit, welche durch wiederholtes Auskochen des Harzes mit Wasser erhalten worden war, vereinigt und mit Äther ausgeschüttelt

Die ätherischen Extracte hinterliessen einen braungefärbten Rückstand, aus einer krystallinischen und einer harzartigen Substanz bestehend.

Von dem grössten Theile des Harzes konnte der Rückstand durch Behandeln mit Calciumcarbonat getrennt werden. Die aus dem gelösten Kalksalze gewonnene Säure wurde durch Auskochen mit Petroleumäther, welcher eine gelbe amorphe Substanz nicht aufnahm, gereinigt und schliesslich aus Wasser umkrystallisirt.

Die Verbindung schmolz zwischen $121-122^{\circ} \mathrm{C}$. und zeigte alle Eig'enschaften der Benzoësäure.

Ein Theil der Säure (im ganzen waren etwa 1.5g erhalten worden) wurde in das Kalksalz umgewandelt, und dieses zur Analyse beniitzt.

$0.4022 g$ Kalksalz verloren bei $130^{\circ}$ C. $0.0634 g$ an Gewicht. $0 \cdot 1683 g$ hinterliessen $0.0336 g$ Calciumoxyd.

$$
\begin{aligned}
& \text { Gefunden: } \\
& \begin{array}{c}
\underbrace{\text { Berechnet für : }}_{\left(\mathrm{C}_{7} \mathrm{H}_{5} \mathrm{O}_{2}\right)_{2} \mathrm{Ca}+3 \mathrm{H}_{2} \mathrm{O} ;\left(\mathrm{C}_{7} \mathrm{H}_{5} \mathrm{O}_{2}\right)_{2} \mathrm{Ca}} \\
\mathrm{H}_{2} \mathrm{O} \ldots 16 \cdot 07 \% ; \\
\mathrm{Ca} . .14 \cdot 18 \%
\end{array} \\
& \mathrm{H}_{2} \mathrm{O} \ldots 15 \cdot 76 \% \\
& \text { Ca..... 14.26\% }
\end{aligned}
$$

1 Es machte sich hiebei der Geruch von Benzaldehyd bemerkbar. 
Ausser der Benzoësäure wird bei der Oxydation des Benzylstrychniliumehlorids das schon erwähnte Harz, das der Menge nach als Hauptproduct erscheint, und eine aus dem Mangansuperoxyd durch schweflige Säure abscheidbare, amorphe, braune Masse $($ ca. $1 \mathrm{~g}$ ) erhalten. Letztere ist im Gegensatze zum Harze in Kalilauge nur theilweise, jedoch wie dieses in Säuren nicht löslich.

Alle Bemühungen, aus diesen Oxydationsproducten wohl charakterisirte Verbindungen abzuscheiden, waren vergeblich. Die relativ bedeutende Menge Benzoësäure, welche bei diesem Oxydationsprocesse gebildet wird, scheint darauf hinzudeuten, dass im Benzylstrychniliumchlorid zuerst eine Abspaltung der Benzylgruppe (beziehungsweise $\mathrm{C}_{7} \mathrm{H}_{6}$ ) erfolgt und die neugebildeten Atomcomplexe sich dann weiterhin mit dem Sauerstoff verbinden.

Laboratorium der I. deutschen k. k. Staatsrealsehule in Prag. 